\title{
Transcranial direct-current stimulation increases extracellular dopamine levels in the rat striatum
}

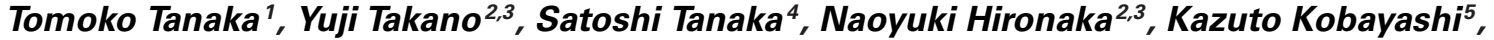 \\ Takashi Hanakawa ${ }^{1,6}$, Katsumi Watanabe ${ }^{7}$ and Manabu Honda ${ }^{1,6 *}$ \\ 1 Department of Functional Brain Research, National Institute of Neuroscience, National Center of Neurology and Psychiatry, Tokyo, Japan \\ 2 Human Information Science Laboratory, NTT Communication Science Laboratories, Kanagawa, Japan \\ ${ }^{3}$ CREST, Japan Science and Technology Agency, Saitama, Japan \\ ${ }^{4}$ Center for Fostering Young and Innovative Researchers, Nagoya Institute of Technology, Aichi, Japan \\ ${ }^{5}$ Department of Molecular Genetics, Institute of Biomedical Sicence, Fukushima Medical University School of Medicine, Fukushima, Japan \\ ${ }^{6}$ Integrative Brain Imaging Center, National Center of Neurology and Psychiatry, Tokyo, Japan \\ 7 Cognitive Science Laboratory, University of Tokyo, Tokyo, Japan
}

\section{Edited by:}

Detlef H. Heck, University of

Tennessee Health Science Center,

USA

\section{Reviewed by:}

Alessandro Stefani, University of Rome, Italy

Masahiko Takada, Kyoto University, Japan

\section{${ }^{*}$ Correspondence:}

Manabu Honda, Department of Functional Brain Research, National Institute of Neuroscience, National Center of Neurology and Psychiatry, 4-1-1 Ogawa-Higashi, Kodaira, Tokyo 187-8502, Japan.

e-mail: honda@ncnp.go.jp
Background: Transcranial direct-current stimulation (tDCS) is a non-invasive procedure that achieves polarity-dependent modulation of neuronal membrane potentials. It has recently been used as a functional intervention technique for the treatment of psychiatric and neurological diseases; however, its neuronal mechanisms have not been fully investigated in vivo.

Objective/Hypothesis: To investigate whether the application of cathodal or anodal tDCS affects extracellular dopamine and serotonin levels in the rat striatum.

Methods: Stimulation and in vivo microdialysis were carried out under urethane anesthesia, and microdialysis probes were slowly inserted into the striatum. After the collection of baseline fractions in the rat striatum, cathodal or anodal tDCS was applied continuously for 10 min with a current intensity of $800 \mu \mathrm{A}$ from an electrode placed on the skin of the scalp. Dialysis samples were collected every 10 min until at least 400 min after the onset of stimulation.

Results: Following the application of cathodal, but not anodal, tDCS for $10 \mathrm{~min}$, extracellular dopamine levels increased for more than $400 \mathrm{~min}$ in the striatum. There were no significant changes in extracellular serotonin levels.

Conclusion: These findings suggest that tDCS has a direct and/or indirect effect on the dopaminergic system in the rat basal ganglia.

Keywords: basal ganglia, dopamine, Parkinson disease, transcranial direct current stimulation, striatum

\section{INTRODUCTION}

Transcranial direct-current stimulation (tDCS) is a non-invasive technique in which a weak DC is used to polarize target brain regions (Nitsche and Paulus, 2000). Several studies have previously shown that tDCS affects motor function and learning in healthy subjects, presumably by changing the neuronal activity of the stimulated site (Wassermann and Grafman, 2005; Tanaka et al., 2009; Bachmann et al., 2010; Fox, 2011; Schambra et al., 2011). It is also effective in patients with psychiatric and neurological diseases, and so has the potential to be used as an adjuvant strategy in the rehabilitation of motor and cognitive deficits caused by neurological disorders (Hummel et al., 2005; Boggio et al., 2006; Fregni et al., 2006; Lefaucheur, 2009; Murphy et al., 2009; Nitsche et al., 2009; Benninger et al., 2010; Tanaka et al., 2011a; Brunoni et al., 2012). In addition, some in vivo animal studies have investigated the behavioral and biological effects of tDCS (Kim et al., 2010; Wachter et al., 2011; Laste et al., 2012). Nevertheless, the mechanisms underlying tDCS are largely unknown, particularly with regard to its effects on the neuronal network.

tDCS directly modulates neuronal membrane potentials beneath the stimulus electrode. However, it might also have a remote or systematic effect on the neuronal circuit. Indeed, increasing evidence from human electrophysiological and neuroimaging studies suggests that tDCS modulates brain activities in cortical or subcortical areas other than the stimulated site (Lang et al., 2005; Bachmann et al., 2010; Antal et al., 2011; Binkofski et al., 2011; Halko et al., 2011), possibly via anatomical connections (Veening et al., 1980; Selemon and Goldman-Rakic, 1985; McGeorge and Faull, 1989). Recently, human experiments using fMRI reported that tDCS can also modulate resting-state functional connectivity in distinct functional networks of the brain (Keeser et al., 2011). Furthermore, it has been demonstrated that an increase of phosphorylation of trkB, which is a receptor of Brain-derived neurotrophic factor (BDNF), was induced by DCS in vitro (Fritsch et al., 2010). These comprehensive 
effects through neuronal networks may be observable at the neurotransmitter level, as well as at the electrophysiological and metabolic levels. Previously, pharmacological approaches based on human drug intake have suggested the involvement of glutamatergic, $\gamma$-aminobutyric acid (GABA)-ergic, and dopaminergic systems in long-term tDCS effects (Liebetanz et al., 2002; Nitsche et al., 2003). These experiments, however, reported modulation of tDCS effects by neurotransmitter operations, and used motor-evoked potentials (MEPs) as outcome measurements. Consequently, they provided indirect measurements of neurotransmitters, which inherently limited the interpretation of results. In the present study, being an important factor in the introduction of a remote network effect, we focused on the change in neurotransmitter levels.

The present study directly measured changes in the extracellular dopamine level in the basal ganglia induced by tDCS using in vivo microdialysis in a rat model. This invasive procedure directly and locally measures compounds, such as extracellular dopamine, via a probe inserted into a target brain region (Navailles et al., 2004; Nitsche et al., 2006). Dopamine transmission in the striatum plays an essential role in the modulation of motor and cognitive symptoms caused by neurological disorders such as Parkinson's disease (PD) (Carlsson, 1972; Fahn, 2003), as well as in learning-induced neuroplasticity in both humans and rats (Adcock et al., 2006; Berridge, 2007; Rossato et al., 2009; Tanaka et al., 2011b). To understand the underlying mechanism of tDCS behavioral effects, we examined whether its application affected the dopaminergic systems in the striatum and showed that it had a direct and/or indirect effect.

\section{MATERIALS AND METHODS}

The experimental protocol was approved by the Animal Care and Use Committee of the National Institute of Neuroscience (National Center of Neurology and Psychiatry, Tokyo, Japan). The experiments were conducted in accordance with the "Official Notification on Animal Experiments" (National Institute of Neuroscience, National Center of Neurology and Psychiatry notification no. 2010004, received 2010). Every effort was made to minimize the number of animals used in the experiments and their suffering.

\section{ANIMALS}

Male nine-week old Sprague Dawley rats (CLEA Japan, Inc., Tokyo, Japan) were housed at a temperature of $23 \pm 1^{\circ} \mathrm{C}$ with a 12-h light/dark cycle (lights-on 08:00-20:00). Food and water were available ad libitum. Twenty-five rats were used for the microdialysis experiment, and 12 were used to investigate tissue damage.

\section{MICRODIALYSIS SURGERY}

Twenty-five rats were divided into the following three groups: cathodal tDCS $(n=7)$, anodal $(n=7)$, and sham $(n=7)$. After at least 3 days of habituation to the animal colony, all rats were intraperitoneally (i.p.) anesthetized with a single shot of urethane ( $1 \mathrm{~g} / \mathrm{kg}$ body weight) and placed in a stereotaxic apparatus. The skull was exposed and a small hole was made using a dental drill. A guide cannula (AG-6; Eicom Corporation, Kyoto,
Japan) was implanted into the striatum $[+1.0 \mathrm{~mm}$ anterior, $+3.5 \mathrm{~mm}$ lateral, and $-4.5 \mathrm{~mm}$ ventral to the bregma according to the stereotaxic atlas of Paxinos and Watson (1998)]. The guide cannula was fixed to the skull with resin dental cement.

\section{tDCS}

The experimental tDCS setup was similar to that reported by Takano et al. (2010). One electrode of the stimulator $(5 \mathrm{~mm} \times$ $5 \mathrm{~mm}$ size) was fixed with surgical tape on the skin above the brain region including the cortex, and positioned in reference to the insertion point of the microdialysis guide cannula (Figure 1A) at an anatomical location roughly corresponding to +2.0 to $+7.0 \mathrm{~mm}$ anterior and +1.0 to $+6.0 \mathrm{~mm}$ lateral to the bregma (Paxinos and Watson, 1998). It was previously reported that the frontal cortex beneath the stimulation electrode had an anatomical connection to the striatum where the microdialysis probe was implanted (Ebrahimi et al., 1992; Gabbott et al., 2005). A second electrode was placed on the animal's neck in a similar way.

Cathodal or anodal tDCS was applied continuously for $10 \mathrm{~min}$ with a current intensity of $800 \mu \mathrm{A}$ from the scalp electrode using a DC stimulator (STG1002; Multi Channel Systems, Germany). Cathodal stimulation was applied for $10 \mathrm{~s}$ to the sham group with a current intensity of $10 \mu \mathrm{A}$ from the scalp electrode. The safety limit of the stimulator was $120 \mathrm{~V}$. The current intensity of $800 \mu \mathrm{A}$, corresponding to a current density of $32.0 \mathrm{~A} / \mathrm{m}^{2}$ in the present setting, was used to maximize the effects of tDCS within the safety limits reported in a previous rat tDCS study using cathodal

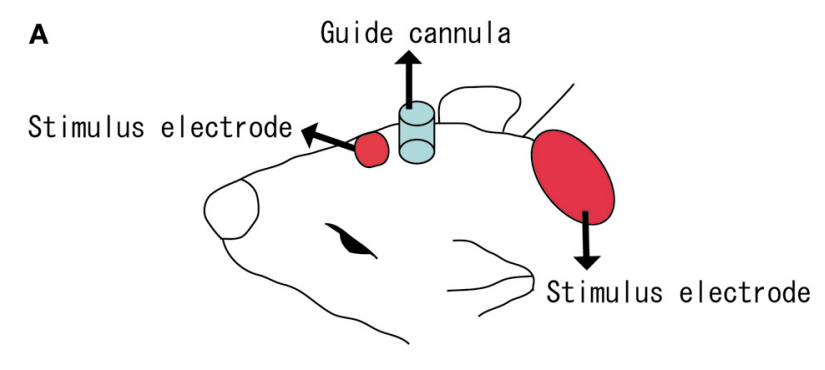

B

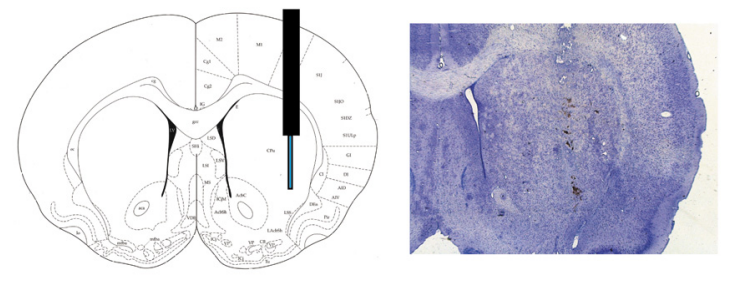

FIGURE 1 | Position of tDCS electrode and microdialysis probe. (A) One electrode (small red square) of the stimulator was fixed to the skin with surgical tape just above the brain region including the cortex. The anatomical location corresponded to +2.0 to $+7.0 \mathrm{~mm}$ anterior and +1.0 to $+6.0 \mathrm{~mm}$ lateral to the bregma. A second electrode (large red ellipse) was placed on the neck. A guide cannula (blue cylinder) was fixed to the skull with resin dental cement; $(\mathbf{B})$ a microdialysis probe was inserted into the striatum $(+1.0 \mathrm{~mm}$ anterior, $+3.5 \mathrm{~mm}$ lateral, and $-4.5 \mathrm{~mm}$ ventral to the bregma). 
stimulation (Liebetanz et al., 2009), although the safety limits of anodal stimulation was not specified.

\section{DOPAMINE AND SEROTONIN MEASUREMENTS}

Stimulation and in vivo microdialysis were carried out under urethane anesthesia. Body temperature and heart rate were monitored and remained at approximately $37^{\circ} \mathrm{C}$ and $350 \mathrm{bpm}$, respectively. No remarkable changes in vigilance were observed by visual inspection of body movement. After surgery, microdialysis probes (AI-6-02; 2-mm membrane length; Eicom Corporation) were slowly inserted into the striatum through the guide cannula (Figure 1B). The probe was perfused continuously at a flow rate of $2 \mu \mathrm{l} / \mathrm{min}$ with artificial cerebrospinal fluid (aCSF) containing $0.9 \mathrm{mM} \mathrm{MgCl}_{2}, 147.0 \mathrm{mM} \mathrm{NaCl}, 4.0 \mathrm{mM} \mathrm{KCl}$, and $1.2 \mathrm{mM} \mathrm{CaCl}_{2}$. The collection and analysis of perfusion solution were performed at $10 \mathrm{~min}$ intervals over the duration of the experiment. The concentrations were judged stable when the fluctuation range of extracellular dopamine and serotonin levels did not exceed $\pm 5 \%$ during six consecutive fractions and when there was not unidirectional fluctuation over more than three fractions (Alex et al., 2005; Kitaichi et al., 2010). After confirming the stability, three fractions were collected from the rat striatum before the application of tDCS as a baseline. Following tDCS, dialysis samples were collected every $10 \mathrm{~min}$ until at least $400 \mathrm{~min}$ after the onset of stimulation.

Dialysis fractions were analyzed using high-performance liquid chromatography (HPLC) with an electrochemical detection (ECD) system (Eicom Corporation). The extracellular serotonin level in the striatum was also measured, as it is known to be associated with the dopamine level (Di Giovanni et al., 2008; Navailles and De Deurwaerdere, 2011). Dopamine and serotonin were separated by a column with a mobile phase containing $99 \% 0.1 \mathrm{M}$ sodium phosphate buffer ( $\mathrm{pH} 6.0$ ), $500 \mathrm{mg} / \mathrm{L}$ sodium 1-decanesulfonate, $50 \mathrm{mg} / \mathrm{L}$ ethylenediaminetetraacetic acid disodium salt (EDTA-2Na), and 1\% methanol. The mobile phase was delivered at a flow rate of $500 \mu \mathrm{l} / \mathrm{min}$. Before every experiment dopamine hydrochloride and serotonin hydrochloride, as the standard reagents, were dissolved in solvent and injected into the HPLC system. Retention times of dopamine and serotonin were calculated from the peaks detected in the chromatograph. During each experiment, the retention time of substances, which were collected from the striatum, was calculated. When the measured retention time matched with those of the standard reagents, collected substances were judged as dopamine or serotonin. All the measurement conditions such as mobile phase for calibration were same as those for actual measurements of rats. Dopamine and serotonin were quantified by calculating the peak areas (Alex et al., 2005; Kitaichi et al., 2010).

To confirm the insertion position of the microdialysis probe after completion of the experiment, all rats were deeply anesthetized with sodium pentobarbital $(50 \mathrm{mg} / \mathrm{kg}$ body weight, i.p.) and perfused through the heart sequentially with $1 \times$ phosphate buffered saline (PBS) followed by $10 \%$ formalin neutral buffer solution. Rat brains were post-fixed and sucrose-substituted at $4^{\circ} \mathrm{C}$, and $20-\mu \mathrm{m}$-thick coronal sections were cut through the striatum ( $-1 \mathrm{~mm}$ to $3 \mathrm{~mm}$ anterior to the bregma) on a cryostat. These were then thaw-mounted on 3-aminopropyltriethoxysilane
(APTS)-coated slides and stained with cresyl violet using standard procedures.

\section{tDCS-INDUCED TISSUE DAMAGE}

Histological examination was performed to determine the effects of tDCS on the brain tissue and skin beneath the scalp electrode. At $24 \mathrm{~h}$ after the application of tDCS, 12 rats (cathodal tDCS and sham groups; $n=6$ per group) were deeply anesthetized with sodium pentobarbital ( $50 \mathrm{mg} / \mathrm{kg}$ body weight, i.p.), and $10-\mu \mathrm{m}$-thick coronal sections of their brains were prepared and stained with cresyl violet using a method similar to that described above. The skins were post-fixed at $4^{\circ} \mathrm{C}$, dehydrated with ethanol and xylene, and paraffin-embedded. Sections (5- $\mu \mathrm{m}$ thick) of rat skin from just below the scalp electrode were cut on a microtome, thaw-mounted on APTS-coated slides, and stained with hematoxylin and eosin (HE) using standard procedures. Morphological changes were then evaluated. To confirm whether tDCS leads to apoptosis, $10-\mu \mathrm{m}$-thick coronal sections were prepared from the same animals and stained with terminal deoxynucleotidyl transferase-mediated biotinylated UTP nick-end labeling (TUNEL) (Kobayashi et al., 2004). To minimize the number of animals used in the experiments, we omitted the anodal tDCS group, which showed no significant changes in dopamine or serotonin levels in the microdialysis experiment (see section Results).

\section{STATISTICAL ANALYSIS}

Data from four rats in which a probe had not been accurately inserted into the striatum (Figure 1B) were excluded from statistical analysis. Extracellular dopamine and serotonin levels were expressed as percentage signal changes from baseline values before tDCS application. Group data are presented as mean \pm standard error $(S E M)$. The statistical significance of differences between groups was assessed by repeated measures analysis of variance (ANOVA) with time (TIME) as a within-subject factor and group (GROUP) as a between-subject factor. This was followed by the post-hoc Bonferroni test using SPSS software (SPSS Inc., Chicago, IL). To investigate whether the time effect differed between groups, we confirmed the "TIME" $\times$ "GROUP" interaction. $P$-values less than $5 \%$ were considered statistically significant. To investigate when the change in dopamine level became prominent, data at each time point were compared with the baseline using the paired $t$-tests. For the same purpose, data from the tDCS group were compared with those in the sham group at each time point separately using the unpaired two-sample $t$-tests.

\section{RESULTS}

Statistical analysis was carried out on rats in the cathodal tDCS $(n=7)$, anodal tDCS $(n=7)$, and sham $(n=7)$ groups. To examine whether cathodal or anodal tDCS affected extracellular dopamine and serotonin levels in the striatum, we investigated the effect of tDCS using in vivo microdialysis. The absolute basal dialysis levels of dopamine and serotonin detected 10 min before the interventions did not differ between the three groups [Table 1; dopamine, $F_{(2,20)}=0.389, p=0.684$; serotonin, $\left.F_{(2,20)}=0.242, p=0.788\right]$. These basal levels compared well with the previous studies (Baumann et al., 2008; Kitaichi 
et al., 2010). Following the application of cathodal tDCS for $10 \mathrm{~min}$, the extracellular dopamine levels continuously increased and this effect lasted for more than $400 \mathrm{~min}$ after the stimulation ceased (Figure 2).

The dopamine time-course in the striatum differed significantly between the three groups [main effect of GROUP, $F_{(1,20)}=27.386, p<0.001$; main effect of TIME, $F_{(44,880)}=$ $0.514, p=0.997$; interaction of GROUP $\times$ TIME, $F_{(2,88)}=$ 4.674, $p<0.001]$. The dopamine increases in the cathodal tDCS group were significantly greater than those in the sham and the anodal tDCS groups ( $p<0.001$ for both, post-hoc test). These increases became statistically prominent $120 \mathrm{~min}$ after the stimulation compared with the pre-intervention period $(p<0.05$, paired $t$-test), and lasted throughout the observation period. Furthermore, the dopamine increases in the cathodal tDCS group were significantly greater than those in the sham group from 120 min after stimulation ( $p<0.05$, unpaired two-sample $t$-test). By contrast, the application of anodal tDCS did not induce significant increases in extracellular dopamine levels.

Table 1 | Absolute basal dialysis levels of dopamine and serotonin.

\begin{tabular}{lll}
\hline Group & Dopamine (nM) & Serotonin (nM) \\
\hline Sham & $0.61 \pm 0.070$ & $0.11 \pm 0.076$ \\
Cathodal tDCS & $0.62 \pm 0.076$ & $0.11 \pm 0.062$ \\
Anodal tDCS & $0.69 \pm 0.071$ & $0.06 \pm 0.012$ \\
\hline
\end{tabular}

Data are presented as the mean \pm SEM.
There were no significant changes in extracellular serotonin levels in the striatum in any group, indicating that they were unaffected by the application of either cathodal or anodal tDCS [Figure 3; main effect of GROUP, $F_{(1,20)}=2.016, p=0.159$; interaction of GROUP $\times$ TIME, $F_{(2,86)}=0.997, p=0.540$, respectively].

One day after the application of tDCS, tissues were dissected and processed for sectioning. The sections through the cortex were used for histological examination with cresyl violet staining. No abnormal findings regarding cellular morphology, such as chromatolysis or atrophied tissue, were observed in the cortex below the scalp electrode of rats in either the cathodal tDCS group or the sham group (Figure 4A). No apoptosis was found in either group (data not shown). The sections through the skin below the scalp electrode were analyzed by HE staining, revealing no abnormalities such as dead tissue, inclusion, or multicell spheroids in either group (Figure 4B).

\section{DISCUSSION}

The main finding of the present study was that cathodal tDCS induced a significant increase in extracellular dopamine levels in the rat striatum, whereas anodal tDCS in the same area had no effect. Although recent magnetic-resonance spectroscopy studies have shown direct effects of tDCS on cortical GABA concentration (Stagg et al., 2009), the effect of tDCS on subcortical neurotransmitters has remained unclear. The present results suggest that tDCS has a direct and/or indirect effect on the dopaminergic system in the basal ganglia. The finding that tDCS affected dopamine but not serotonin is consistent with a

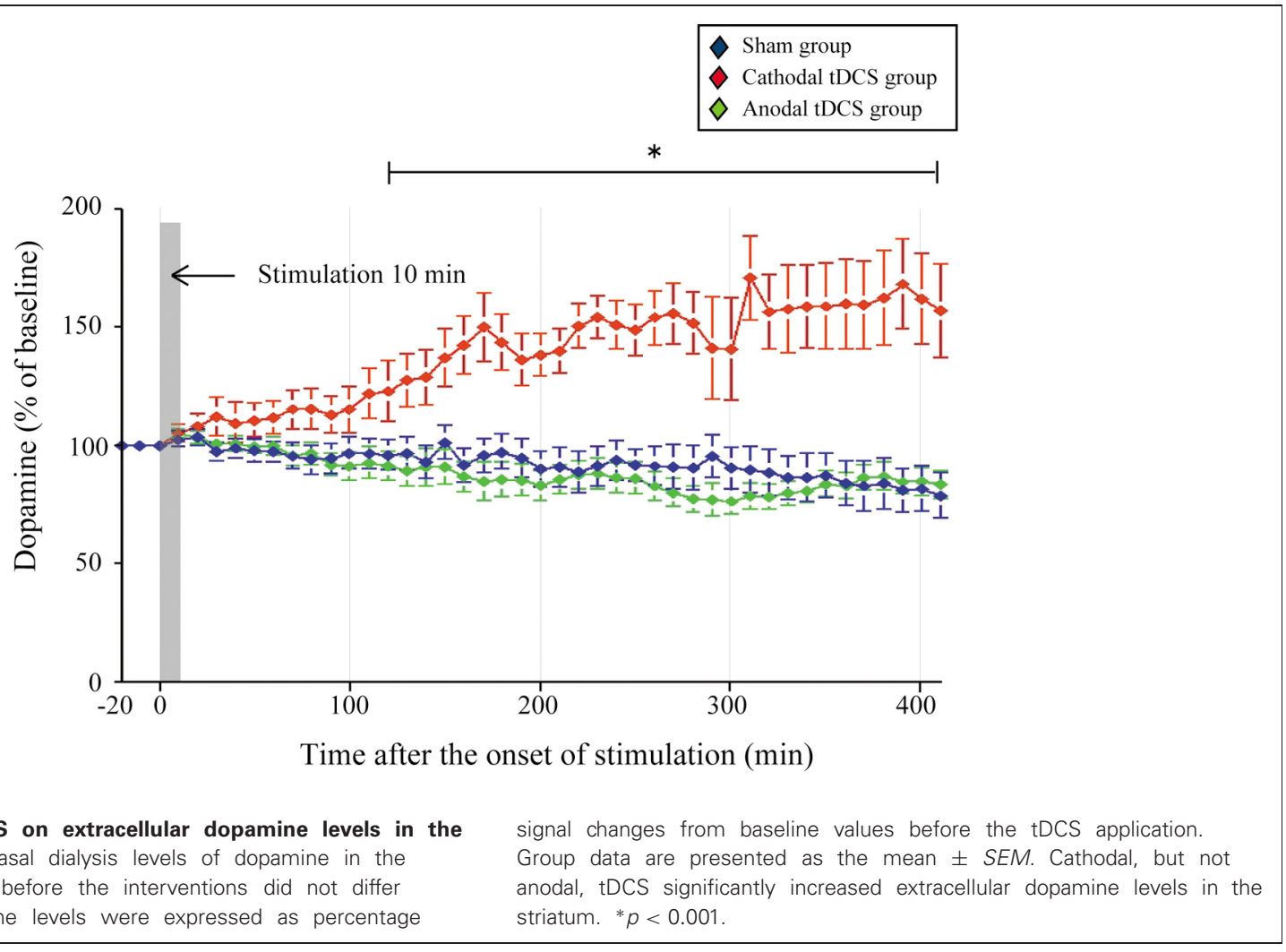



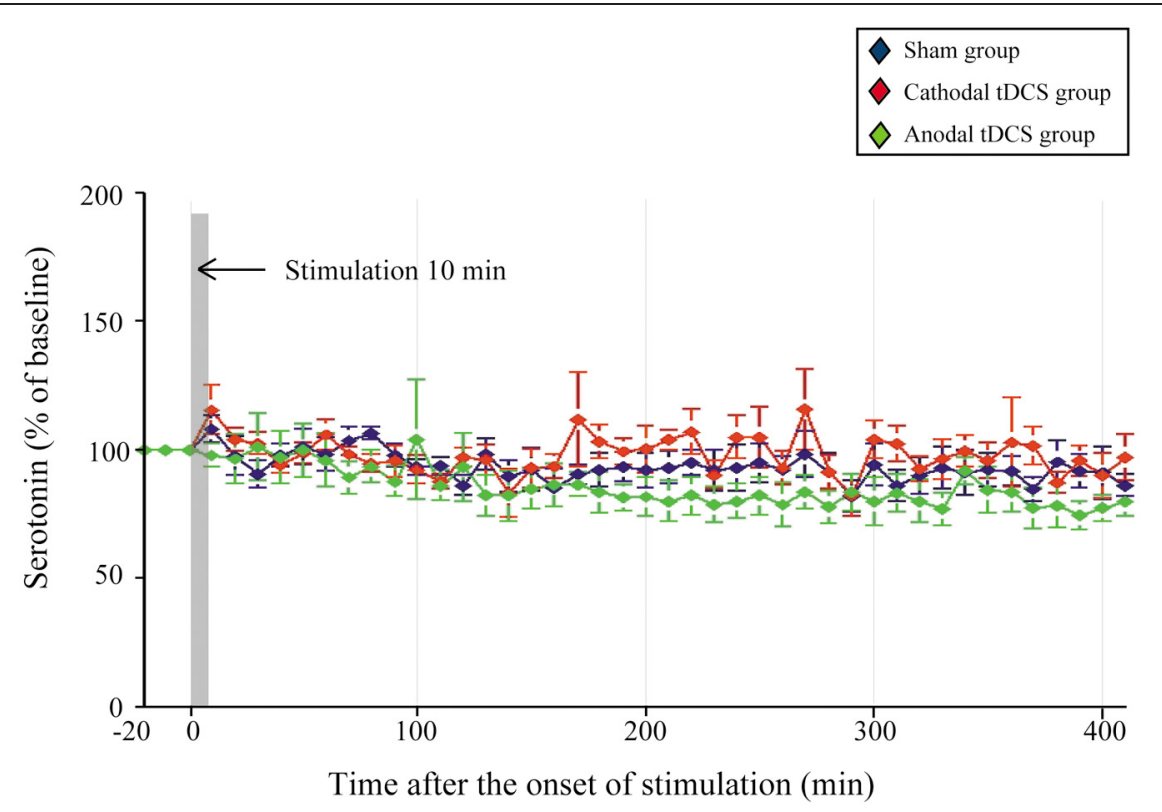

FIGURE 3 | Effect of tDCS on extracellular serotonin levels in the striatum. The absolute basal dialysis levels of serotonin in the striatum detected $10 \mathrm{~min}$ before the interventions did not differ between groups. Serotonin levels were expressed as percentage signal changes from baseline values before the tDCS application. Group data are presented as the mean \pm SEM. None of the tDCS applications significantly affected extracellular serotonin levels. previous study that reported no changes in the rat serotonergic system following repetitive transcranial magnetic stimulation (rTMS) (Kanno et al., 2004). As the tDCS methods used here did not damage tissue in the cortex or the skin below the scalp electrode, it is unlikely that the rise in dopamine was induced by non-specific cellular injury.

\section{INTERPRETATION OF THE CATHODAL tDCS EFFECT}

Several studies have shown that diverse areas of the cerebral cortex, including the sensory, motor, and associated regions, project to the subcortical regions, including the striatum (McGeorge and Faull, 1989; Lang et al., 2005; Halko et al., 2011). Furthermore, it has been reported that descending pathways from the frontal cortex to the striatum modulate a release of dopamine in subcortical areas in both animal and human experiments (Murase et al., 1993; Taber and Fibiger, 1995; Karreman and Moghaddam, 1996). Considering that $\mathrm{tDCS}$ has been found to improve symptoms of PD in both animal and human experiments (Boggio et al., 2006; Fregni et al., 2006; Benninger et al., 2010; Gruner et al., 2010; Li et al., 2011), it is plausible that tDCS could directly and/or indirectly affect the extracellular dopamine levels in the striatum.

However, our finding that cathodal tDCS, but not anodal, increased extracellular dopamine levels is contradictory to our prediction and inconsistent with previous studies. For example, cathodal tDCS has been thought to induce suppression of motor function and learning by inhibiting neuronal excitability of the cortex (Murphy et al., 2009; Nitsche et al., 2009; Bachmann et al., 2010; Benninger et al., 2010; Fox, 2011; Schambra et al., 2011; Tanaka et al., 2011a). In addition, rTMS has been suggested to induce increased extracellular dopamine levels in the striatum by facilitating neuronal excitability of the cortex (Keck et al., 2002;
Strafella et al., 2003; Ohnishi et al., 2004). We acknowledge that the present finding is contradictory to such studies.

To our knowledge, this is the first report that directly measured extracellular dopamine levels in the striatum-induced by tDCS. Although the mechanism underlying the long-lasting effect of cathodal tDCS observed in the present study has not yet been determined, we offer the following speculations. It has been widely accepted that cathodal tDCS decreases cortical neuronal activity (Bindman et al., 1964; Purpura and McMurtry, 1965; Fritsch et al., 2010). Meanwhile, a recent report showed that cathodal, but not anodal, tDCS induced paired pulse facilitation in the somatosensory cortex of rabbits following stimulation of the ventroposterior medial thalamic nucleus (Marquez-Ruiz et al., 2012). This study suggests that cathodal tDCS may specifically facilitate synaptic plasticity. Furthermore, cathodal, but not anodal, tDCS was shown to facilitate working memory and skill learning 21 days after treatment, implying that cathodal tDCS might facilitate long-term homeostatic cortical metaplasticity (Dockery et al., 2011). Although the cathodal tDCS may instantaneously decrease neuronal activity beneath the stimulus electrode, these findings suggest that cathodal tDCS may specifically induce a long-term plastic change thorough some metabolic changes, such as BDNF release (Fritsch et al., 2010). We speculate that the increase of dopamine release in the striatum may contribute to such long-term plasticity.

Alternatively, the cortico-basal ganglia neural circuit contains both excitatory and inhibitory projections (Alexander and Crutcher, 1990; Nambu et al., 2000) in an intrinsic neuronal network. Therefore, we must also consider the possibility that cathodal tDCS affects extracellular dopamine levels in the striatum through an inhibitory circuit. 

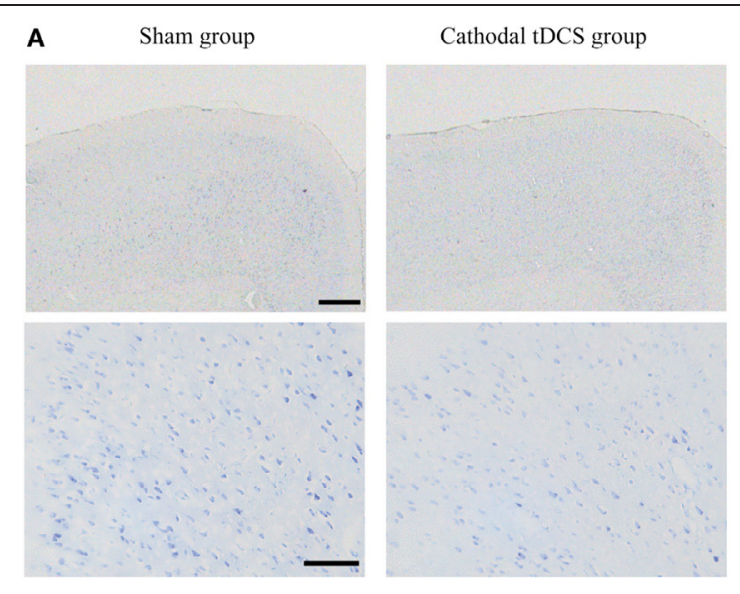

B
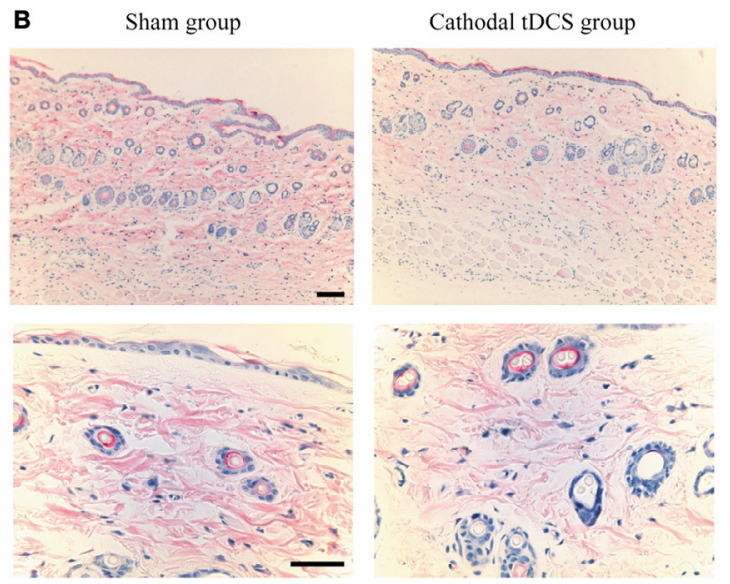

FIGURE 4 | Histological examination following tDCS. (A) Cresyl violet staining of the brain tissue below the scalp electrode in the sham group (left) and the cathodal tDCS group (right) $24 \mathrm{~h}$ after tDCS application. Scale bars: $1 \mathrm{~mm}$ for upper panels, $100 \mu \mathrm{m}$ for lower panels; (B) HE staining of the skin below the scalp electrode in the sham group (left) and the cathodal tDCS group (right) $24 \mathrm{~h}$ after tDCS application. Scale bars: $100 \mu \mathrm{m}$ for upper panels, $50 \mu \mathrm{m}$ for lower panels. No morphological change was observed in brain tissue and skin.

\section{EXPERIMENTAL LIMITATIONS}

General anesthesia affects brain metabolism, neuronal activity, and response to sensory stimuli (Buzsaki et al., 1983; Friedberg et al., 1999); it is thus conceivable that the present results were partly influenced by anesthesia. However, this is unlikely to be the only reason for the cathodal tDCS-specific dopamine increase, as the anesthetic procedure was common to all stimulation conditions. Moreover, in a previous study, alteration of extracellular dopamine levels in the striatum induced by medial forebrain

\section{REFERENCES}

Adcock, R. A., Thangavel, A., WhitfieldGabrieli, S., Knutson, B., and Gabrieli, J. D. (2006). Rewardmotivated learning: mesolimbic activation precedes memory formation. Neuron 50, 507-517.

Alex, K. D., Yavanian, G. J., McFarlane, H. G., Pluto, C. P., and Pehek, E.
A. (2005). Modulation of dopamine release by striatal 5-HT2C receptors. Synapse 55, 242-251.

Alexander, G. E., and Crutcher, M. D. (1990). Functional architecture of basal ganglia circuits: neural substrates of parallel processing. Trends Neurosci. 13, 266-271.

Antal, A., Polania, R., Schmidt-Samoa, C., Dechent, P., and Paulus, W.

bundle stimulation did not differ between urethane-anesthetized and awake animals (Tepper et al., 1991).

The contamination effect induced by the tDCS reference electrode on the neck should be considered. Although the electrodecurrent density in this region was lower and less effective, and there is no brain tissue beneath the tDCS reference electrode on the neck, it was unclear exactly how the current flowed between the two electrodes in the present experimental setup. Furthermore, the current delivered by the stimulation electrode might have reached the striatum through the guide cannula, even though the dental cement used to fix it was an electrical insulator. To rule out this possibility, extracellular dopamine levels should be measured using a non-invasive technique such as positron-emission tomography.

Though polarity of their affective stimulation electrode differed from that of the present study, some reports have shown that tDCS improves PD symptoms in both animals and humans (Boggio et al., 2006; Fregni et al., 2006; Benninger et al., 2010; Gruner et al., 2010; Li et al., 2011). An underlying mechanism of such clinical effects could be an increase in extracellular dopamine levels in the striatum induced by tDCS. However, it is as of yet unclear whether $150 \%$ of basal dopamine level is clinically relevant and if tDCS will increase dopamine levels in patients whose dopaminergic neurons have degenerated. Therefore, we recommend caution in correlating the present finding to clinical application of $\mathrm{tDCS}$.

In conclusion, we demonstrated that cathodal tDCS increased extracellular dopamine levels in the rat striatum. This suggests that tDCS has a direct and/or indirect effect on the dopaminergic system in the subcortical area. Further work to determine the mechanism underlying tDCS effects on cortical-basal ganglia functions could benefit our understanding of learninginduced neuroplasticity and the development of new clinical interventions.

\section{AUTHOR CONTRIBUTIONS}

Tomoko Tanaka, Yuji Takano, Satoshi Tanaka, Naoyuki Hironaka, Kazuto Kobayashi, Takashi Hanakawa, Katsumi Watanabe, and Manabu Honda designed the research; Tomoko Tanaka performed the research; Tomoko Tanaka analyzed the data; Tomoko Tanaka, Satoshi Tanaka, and Manabu Honda wrote the paper.

\section{ACKNOWLEDGMENTS}

We thank S. Shimojo and T. Nishikawa for comments on this work. This work was partly supported by JSPS KAKENHI Grant Number 22240049 and by MEXT KAKENHI Grant Number 22135008 for Manabu Honda and by JST for Naoyuki Hironaka and Katsumi Watanabe.

(2011). Transcranial direct current stimulation over the primary motor cortex during fMRI. Neuroimage 55, 590-596.

Bachmann, C. G., Muschinsky, S., Nitsche, M. A., Rolke, R., Magerl, W., Treede, R. D., et al. (2010). Transcranial direct current stimulation of the motor cortex induces distinct changes in thermal and mechanical sensory percepts. Clin. Neurophysiol. 121, 2083-2089.

Baumann, M. H., Clark, R. D., and Rothman, R. B. (2008). Locomotor stimulation produced by 3,4 methylenedioxymethamphetamine (MDMA) is correlated with dialysate levels of serotonin and dopamine in rat brain. Pharmacol. Biochem. Behav. 90, 208-217. 
Benninger, D. H., Lomarev, M., Lopez, G., Wassermann, E. M., Li, X., Considine, E., et al. (2010). Transcranial direct current stimulation for the treatment of Parkinson's disease. J. Neurol. Neurosurg. Psychiatry 81, 1105-1111.

Berridge, K. C. (2007). The debate over dopamine's role in reward: the case for incentive salience. Psychopharmacology (Berl.) 191, 391-431.

Bindman, L. J., Lippold, O. C., and Redfearn, J. W. (1964). The action of brief polarizing currents on the cerebral cortex of the rat (1) during current flow and (2) in the production of longlasting after-effects. J. Physiol. 172, 369-382.

Binkofski, F., Loebig, M., Jauch-Chara, K., Bergmann, S., Melchert, U. H., Scholand-Engler, H. G., et al. (2011). Brain energy consumption induced by electrical stimulation promotes systemic glucose uptake. Biol. Psychiatry 70, 690-695.

Boggio, P. S., Ferrucci, R., Rigonatti, S. P., Covre, P., Nitsche, M., PascualLeone, A., et al. (2006). Effects of transcranial direct current stimulation on working memory in patients with Parkinson's disease. J. Neurol. Sci. 249, 31-38.

Brunoni, A. R., Nitsche, M. A., Bolognini, N., Bikson, M., Wagner, T., Merabet, L., et al. (2012). Clinical research with transcranial direct current stimulation (tDCS): challenges and future directions. Brain Stimul. 5, 175-195.

Buzsaki, G., Leung, L. W., and Vanderwolf, C. H. (1983). Cellular bases of hippocampal EEG in the behaving rat. Brain Res. 287, 139-171.

Carlsson, A. (1972). Biochemical and pharmacological aspects of Parkinsonism. Acta Neurol. Scand. Suppl. 51, 11-42.

Di Giovanni, G., Di Matteo, V., Pierucci, M., and Esposito, E. (2008). Serotonin-dopamine interaction: electrophysiological evidence. Prog. Brain Res. 172, 45-71.

Dockery, C. A., Liebetanz, D., Birbaumer, N., Malinowska, M., and Wesierska, M. J. (2011). Cumulative benefits of frontal transcranial direct current stimulation on visuospatial working memory training and skill learning in rats. Neurobiol. Learn. Mem. 96, 452-460.

Ebrahimi, A., Pochet, R., and Roger, M. (1992). Topographical organization of the projections from physiologically identified areas of the motor cortex to the striatum in the rat. Neurosci. Res. 14, 39-60.

Fahn, S. (2003). Description of Parkinson's disease as a clinical syndrome. Ann. N.Y. Acad. Sci. 991, $1-14$.

Fox, D. (2011). Neuroscience: brain buzz. Nature 472, 156-158.

Fregni, F., Boggio, P. S., Santos, M. C., Lima, M., Vieira, A. L., Rigonatti, S. P., et al. (2006). Noninvasive cortical stimulation with transcranial direct current stimulation in Parkinson's disease. Mov. Disord. 21, 1693-1702.

Friedberg, M. H., Lee, S. M., and Ebner, F. F. (1999). Modulation of receptive field properties of thalamic somatosensory neurons by the depth of anesthesia. J. Neurophysiol. 81, 2243-2252.

Fritsch, B., Reis, J., Martinowich, K., Schambra, H. M., Ji, Y., Cohen, L. G., et al. (2010). Direct current stimulation promotes BDNFdependent synaptic plasticity: potential implications for motor learning. Neuron 66, 198-204.

Gabbott, P. L., Warner, T. A., Jays, P. R., Salway, P., and Busby, S. J. (2005). Prefrontal cortex in the rat: projections to subcortical autonomic, motor, and limbic centers. J. Comp. Neurol. 492, 145-177.

Gruner, U., Eggers, C., Ameli, M., Sarfeld, A. S., Fink, G. R., and Nowak, D. A. (2010). $1 \mathrm{~Hz}$ rTMS preconditioned by tDCS over the primary motor cortex in Parkinson's disease: effects on bradykinesia of arm and hand. J. Neural Transm. $117,207-216$.

Halko, M. A., Datta, A., Plow, E. B., Scaturro, J., Bikson, M., and Merabet, L. B. (2011). Neuroplastic changes following rehabilitative training correlate with regional electrical field induced with tDCS. Neuroimage 57, 885-891.

Hummel, F., Celnik, P., Giraux, P., Floel, A., Wu, W. H., Gerloff, C., et al. (2005). Effects of non-invasive cortical stimulation on skilled motor function in chronic stroke. Brain 128, 490-499.

Kanno, M., Matsumoto, M., Togashi, H., Yoshioka, M., and Mano, Y. (2004). Effects of acute repetitive transcranial magnetic stimulation on dopamine release in the rat dorsolateral striatum. J. Neurol. Sci. 217, 73-81.

Karreman, M., and Moghaddam, B. (1996). The prefrontal cortex regulates the basal release of dopamine in the limbic striatum: an effect mediated by ventral tegmental area. J. Neurochem. 66, 589-598.

Keck, M. E., Welt, T., Muller, M. B. Erhardt, A., Ohl, F., Toschi, N., et al. (2002). Repetitive transcranial magnetic stimulation increases the release of dopamine in the mesolimbic and mesostriatal system. Neuropharmacology 43 101-109.

Keeser, D., Meindl, T., Bor, J., Palm, U., Pogarell, O., Mulert, C., et al. (2011). Prefrontal transcranial direct current stimulation changes connectivity of resting-state networks during fMRI. J. Neurosci. 31, 15284-15293.

Kim, S. J., Kim, B. K., Ko, Y. J., Bang, M. S., Kim, M. H., and Han, T. R. (2010). Functional and histologic changes after repeated transcranial direct current stimulation in rat stroke model. J. Korean Med. Sci. 25, 1499-1505.

Kitaichi, Y., Inoue, T., Nakagawa, S. Boku, S., Kakuta, A., Izumi, T., et al. (2010). Sertraline increases extracellular levels not only of serotonin, but also of dopamine in the nucleus accumbens and striatum of rats. Eur. J. Pharmacol. 647, 90-96.

Kobayashi, K., Takahashi, M. Matsushita, N., Miyazaki, J., Koike, M., Yaginuma, H., et al. (2004). Survival of developing motor neurons mediated by Rho GTPase signaling pathway through Rho-kinase. J. Neurosci. 24 3480-3488.

Lang, N., Siebner, H. R., Ward, N. S. Lee, L., Nitsche, M. A., Paulus, W. et al. (2005). How does transcranial DC stimulation of the primary motor cortex alter regional neuronal activity in the human brain? Eur. J. Neurosci. 22, 495-504.

Laste, G., Caumo, W., Adachi, L. N., Rozisky, J. R., De Macedo, I. C., Filho, P. R., et al. (2012). Aftereffects of consecutive sessions of transcranial direct current stimulation (tDCS) in a rat model of chronic inflammation. Exp. Brain Res. 221, 75-83.

Lefaucheur, J. P. (2009). Methods of therapeutic cortical stimulation. Neurophysiol. Clin. 39, 1-14.

Li, Y., Tian, X., Qian, L., Yu, X., and Jiang, W. (2011). Anodal transcranial direct current stimulation relieves the unilateral bial of a rat model of Parikinson's disease. Conf. Proc. IEEE Eng. Med. Biol. Soc. 2011 , 765-768.

Liebetanz, D., Koch, R., Mayenfels, S. Konig, F., Paulus, W., and Nitsche, M. A. (2009). Safety limits of cathodal transcranial direct current stimulation in rats. Clin. Neurophysiol. 120, 1161-1167.

Liebetanz, D., Nitsche, M. A. Tergau, F., and Paulus, W. (2002). Pharmacological approach to the mechanisms of transcranial DCstimulation-induced after-effects of human motor cortex excitability. Brain 125, 2238-2247.

Marquez-Ruiz, J., Leal-Campanario, R., Sanchez-Campusano, R. Molaee-Ardekani, B., Wendling, F., Miranda, P. C., et al. (2012). Transcranial direct-current stimulation modulates synaptic mechanisms involved in associative learning in behaving rabbits. Proc. Natl. Acad. Sci. U.S.A. 109, 6710-6715.

McGeorge, A. J., and Faull, R. L. (1989). The organization of the projection from the cerebral cortex to the striatum in the rat. Neuroscience 29, 503-537.

Murase, S., Grenhoff, J., Chouvet, G., Gonon, F. G., and Svensson, T. H. (1993). Prefrontal cortex regulates burst firing and transmitter release in rat mesolimbic dopamine neurons studied in vivo. Neurosci. Lett. 157, 53-56.

Murphy, D. N., Boggio, P., and Fregni, F. (2009). Transcranial direct current stimulation as a therapeutic tool for the treatment of major depression: insights from past and recent clinical studies. Curr. Opin. Psychiatry 22, 306-311.

Nambu, A., Tokuno, H., Hamada, I., Kita, H., Imanishi, M., Akazawa, T., et al. (2000). Excitatory cortical inputs to pallidal neurons via the subthalamic nucleus in the monkey. J. Neurophysiol. 84, 289-300.

Navailles, S., and De Deurwaerdere, P. (2011). Presynaptic control of serotonin on striatal dopamine function. Psychopharmacology (Berl.) 213, 213-242.

Navailles, S., De Deurwaerdere, P., Porras, G., and Spampinato, U. (2004). In vivo evidence that 5HT2C receptor antagonist but not agonist modulates cocaineinduced dopamine outflow in the rat nucleus accumbens and striatum. Neuropsychopharmacology 29, 319-326.

Nitsche, M. A., Boggio, P. S., Fregni, F., and Pascual-Leone, A. (2009). Treatment of depression with transcranial direct current stimulation (tDCS): a review. Exp. Neurol. 219, 14-19.

Nitsche, M. A., Fricke, K., Henschke, U., Schlitterlau, A., Liebetanz, D., Lang, N., et al. (2003). Pharmacological modulation of cortical excitability shifts induced by transcranial direct current stimulation in humans. J. Physiol. 553, 293-301.

Nitsche, M. A., Lampe, C., Antal, A., Liebetanz, D., Lang, N., Tergau, F., 
et al. (2006). Dopaminergic modulation of long-lasting direct currentinduced cortical excitability changes in the human motor cortex. Eur. J. Neurosci. 23, 1651-1657.

Nitsche, M. A., and Paulus, W. (2000). Excitability changes induced in the human motor cortex by weak transcranial direct current stimulation. J. Physiol. 527(Pt 3), 633-639.

Ohnishi, T., Hayashi, T., Okabe, S., Nonaka, I., Matsuda, H., Iida, H., et al. (2004). Endogenous dopamine release induced by repetitive transcranial magnetic stimulation over the primary motor cortex: an [11C]raclopride positron emission tomography study in anesthetized macaque monkeys. Biol. Psychiatry $55,484-489$.

Paxinos, G., and Watson, C. (1998). The Rat Brain in Stereotaxic Coordinates. San Diego, CA: Academic Press.

Purpura, D. P., and McMurtry, J. G. (1965). Intracellular activities and evoked potential changes during polarization of motor cortex. J. Neurophysiol. 28, 166-185.

Rossato, J. I., Bevilaqua, L. R., Izquierdo, I., Medina, J. H., and Cammarota, M. (2009). Dopamine controls persistence of long-term memory storage. Science 325, 1017-1020.

Schambra, H. M., Abe, M., Luckenbaugh, D. A., Reis, J., Krakauer, J. W., and Cohen, L. G. (2011). Probing for hemispheric specialization for motor skill learning: a transcranial direct current stimulation study. J. Neurophysiol. 106, 652-661.

Selemon, L. D., and Goldman-Rakic, P. S. (1985). Longitudinal topography and interdigitation of corticostriatal projections in the rhesus monkey. J. Neurosci. 5, 776-794.

Stagg, C. J., Best, J. G., Stephenson, M. C., O'Shea, J., Wylezinska, M., Kincses, Z. T., et al. (2009). Polarity-sensitive modulation of cortical neurotransmitters by transcranial stimulation. J. Neurosci. 29, 5202-5206.

Strafella, A. P., Paus, T., Fraraccio, M., and Dagher, A. (2003). Striatal dopamine release induced by repetitive transcranial magnetic stimulation of the human motor cortex. Brain 126, 2609-2615.

Taber, M. T., and Fibiger, H. C. (1995). Electrical stimulation of the prefrontal cortex increases dopamine release in the nucleus accumbens of the rat: modulation by metabotropic glutamate receptors. J. Neurosci. 15, 3896-3904.

Takano, Y., Yokawa, T., Masuda, A., Niimi, J., Tanaka, S., and Hironaka, N. (2010). A rat model for measuring the effectiveness of transcranial direct current stimulation using fMRI. Neurosci. Lett. 491, 40-43.

Tanaka, S., Hanakawa, T., Honda, M., and Watanabe, K. (2009).
Enhancement of pinch force in the lower leg by anodal transcranial direct current stimulation. Exp. Brain Res. 196, 459-465.

Tanaka, S., Sandrini, M., and Cohen, L. G. (2011a). Modulation of motor learning and memory formation by non-invasive cortical stimulation of the primary motor cortex. Neuropsychol. Rehabil. 21, 650-675.

Tanaka, T., Kai, N., Kobayashi, K., Takano, Y., and Hironaka, N. (2011b). Up-regulation of dopamine D1 receptor in the hippocampus after establishment of conditioned place preference by cocaine. Neuropharmacology 61, 842-848.

Tepper, J. M., Creese, I., and Schwartz, D. H. (1991). Stimulus-evoked changes in neostriatal dopamine levels in awake and anesthetized rats as measured by microdialysis. Brain Res. 559, 283-292.

Veening, J. G., Cornelissen, F. M., and Lieven, P. A. (1980). The topical organization of the afferents to the caudatoputamen of the rat. A horseradish peroxidase study. Neuroscience 5, 1253-1268.

Wachter, D., Wrede, A., SchulzSchaeffer, W., Taghizadeh-Waghefi, A., Nitsche, M. A., Kutschenko, A., et al. (2011). Transcranial direct current stimulation induces polarity-specific changes of cortical blood perfusion in the rat. Exp. Neurol. 227, 322-327.

Wassermann, E. M., and Grafman, J. (2005). Recharging cognition with DC brain polarization. Trends Cogn. Sci. 9, 503-505.

Conflict of Interest Statement: The authors declare that the research was conducted in the absence of any commercial or financial relationships that could be construed as a potential conflict of interest.

Received: 04 December 2012; accepted: 16 March 2013; published online: 11 April 2013.

Citation: Tanaka T, Takano Y, Tanaka S, Hironaka N, Kobayashi K, Hanakawa T, Watanabe $K$ and Honda M (2013) Transcranial direct-current stimulation increases extracellular dopamine levels in the rat striatum. Front. Syst. Neurosci. 7:6. doi: 10.3389/fnsys.2013.00006

Copyright (C) 2013 Tanaka, Takano, Tanaka, Hironaka, Kobayashi, Hanakawa, Watanabe and Honda. This is an open-access article distributed under the terms of the Creative Commons Attribution License, which permits use, distribution and reproduction in other forums, provided the original authors and source are credited and subject to any copyright notices concerning any third-party graphics etc. 